Eiji Hanaoka • Toshinori Ozaki • Miki Ohira

Yohko Nakamura • Mikio Suzuki • Ei-ichi Takahashi

Hideshige Moriya • Akira Nakagawara

Shigeru Sakiyama

\title{
Molecular cloning and expression analysis of the human DA41 gene and its mapping to chromosome 9q21.2-q21.3
}

\begin{abstract}
DA41 was previously identified as one of the DAN-binding proteins, via a yeast-based two-hybrid screening strategy. In the present study, we cloned a human homolog of $D A 41$ cDNA. Structural analysis revealed that human DA41 cDNA consisted of 2,861 nucleotides in length and encoded a protein of 589 amino acids, with a predicted molecular mass of $62.4 \mathrm{kDa}$. Human DA41 exhibited an $86 \%$ amino acid sequence identity to rat DA41, indicating the evolutionarily conserved structure and function of DA41. A database search for DA41-related protein(s) identified mouse PLIC-1, PLIC-2, frog XDRP1, and yeast DSK2. DA41 and each DA41-related protein contain a ubiquitin-like domain in their amino-terminal regions. DA41 was expressed ubiquitously in adult human tissues, with relatively higher levels in pituitary gland, adrenal gland, kidney, thymus, and placenta. Fluorescence in situ hybridization (FISH) revealed that $D A 41$ was mapped to human chromosome 9q21.2-q21.3, a position overlapping the candidate tumor suppressor locus for bladder cancer.
\end{abstract}

Key words Chromosome 9q · DA41 · DAN · FISH · Ubiquitin-like protein

E. Hanaoka $\cdot$ T. Ozaki $(\bowtie) \cdot$ M. Ohira $\cdot$ Y. Nakamura

A. Nakagawara $\cdot$ S. Sakiyama

Division of Biochemistry, Chiba Cancer Center Research Institute, 666-2 Nitona, Chuo-ku, Chiba 260-8717, Japan

Tel. +81-43-264-5431; Fax +81-43-262-8680

e-mail: tozaki@biolab.kazusa.or.jp

M. Suzuki · E. Takahashi

Otsuka GEN Research Institute, Otsuka Pharmaceutical Co.,

Ltd., Tokushima, Japan

E. Hanaoka $\cdot$ H. Moriya

Department of Orthopedic Surgery, Chiba University School of

Medicine, Chiba, Japan

The sequence data described in this article have been deposited with the EMBL/GenBank/DDBJ Data libraries under Accession no. $\mathrm{AB} 035275$

\section{Introduction}

The $D A N$ gene was originally cloned as one of the genes whose expression was significantly reduced in rat fibroblast $3 \mathrm{Y} 1$ cells transformed with Rous sarcoma virus (SR-3Y1) (Ozaki and Sakiyama 1993). The expression of $D A N$ was also downregulated in rodent fibroblasts transformed with a variety of oncogenes (Ozaki and Sakiyama 1993; Ozaki et al. 1996). Overexpression of $D A N$ in SR-3Y1 cells markedly suppressed the transformed phenotypes of the recipient cells, indicating the growth- and tumor-suppressive properties of DAN protein (Ozaki and Sakiyama 1994). Interestingly, DAN is a secreted glycoprotein (Nakamura et al. 1997; Stanley et al. 1998) and possesses a characteristic cysteineknot structure common to the DAN/Cerberus family, which includes Gremlin/Drm (Hsu et al. 1998). Like DAN, the expression of Drm was significantly decreased in various transformed cells and its gene product showed a growthsuppressive activity in vitro (Topol et al. 1997). Recently, Hsu et al. (1998) demonstrated that the DAN/Cerberus family can interact with BMP2 and block BMP2 signalling in Xenopus early embryos. Similar results were also obtained in mammalian cell systems (Pearce et al. 1999).

DA41 was initially identified as a new cellular protein which can associate with DAN, in a study that used a yeast two-hybrid screening of an adult rat lung cDNA library (Ozaki et al. 1997). The interaction between DAN and DA41 was mediated through the amino-terminal domain and the cysteine-knot region of DAN. The expression of $D A 41$ was regulated in a cell cycle-dependent manner. In the present study, we performed cDNA cloning and characterization of human $D A 41$, including its expression and its chromosomal mapping.

\section{Methods, results, and discussion}

In order to isolate a full-length human homolog of $D A 41$ cDNA, we screened a human nigra cDNA library (approximately $5 \times 10^{6}$ independent phage clones), using a radio- 
labeled full-length rat $D A 41 \mathrm{cDNA}$ as a probe. Among seven positives, we picked up two independent clones ( $\lambda 41-7$ and $\lambda 41-9)$ which harbored the longer cDNA inserts. Because the putative initiation codon was not located within the nucleotide sequences of these cDNA clones, the 5'-region of DA41-7 cDNA was radiolabeled and used to screen a human lung cDNA library (approximately $5 \times 10^{6}$ recombinants). This screening yielded four independent positive signals. Sequence analysis revealed that the clone 241-11 contained a possible initiation codon (at position +1 ), which fulfilled Kozak's criteria for an initiation codon (Kozak 1987), and was preceded by an in-frame stop codon at position -30 . The assembled human DA41 cDNA consisted of 2,861 nucleotides and contained a single open reading frame of 1,767 nucleotides. The $3^{\prime}$-untranslated region (UTR) contained a consensus polyadenylation signal

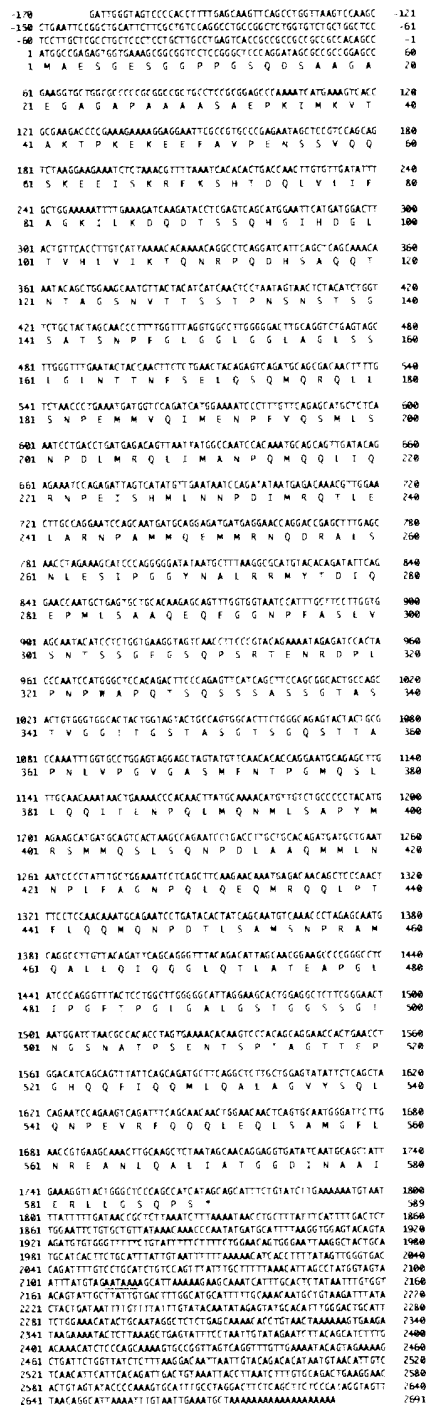

Fig. 1. The nucleotide and deduced amino acid sequences of human $D A 41$ cDNA. The position of the stop codon is indicated by the asterisk and the putative polyadenylation signal is underlined. The nucleotide sequence reported here will appear in EMBL/GenBank/DDBJ Data libraries under Accession no. AB035275
AATAAA at position $+2,111$ and a poly(A) sequence at the end (Fig. 1). The $D A 41$ gene possibly exists as a single copy in the human genome, as examined by genomic Southern analysis (data not shown). Human DA41 encoded a protein consisting of 589 amino acid residues with a predicted molecular mass of $62.4 \mathrm{kDa}$. Human DA41 exhibited $86 \%$ identity to rat DA41 at the protein level (Fig. 2).

A recent database search for DA41-related protein(s) identified mouse PLIC-1 and PLIC-2 (Wu et al. 1999), frog XDRP1 (Funakoshi et al. 1999), and yeast DSK2 (Biggins et al. 1996). PLIC-1 and PLIC-2 are closely related in sequence (higher than $80 \%$ sequence identity between them). Human DA41 exhibited $81 \%, 61 \%, 57 \%$, and $20 \%$ amino acid sequence identity to PLIC-1, PLIC-2, XDRP1, and DSK2, respectively (Fig. 2). PLIC-1 and PLIC-2 were identified to

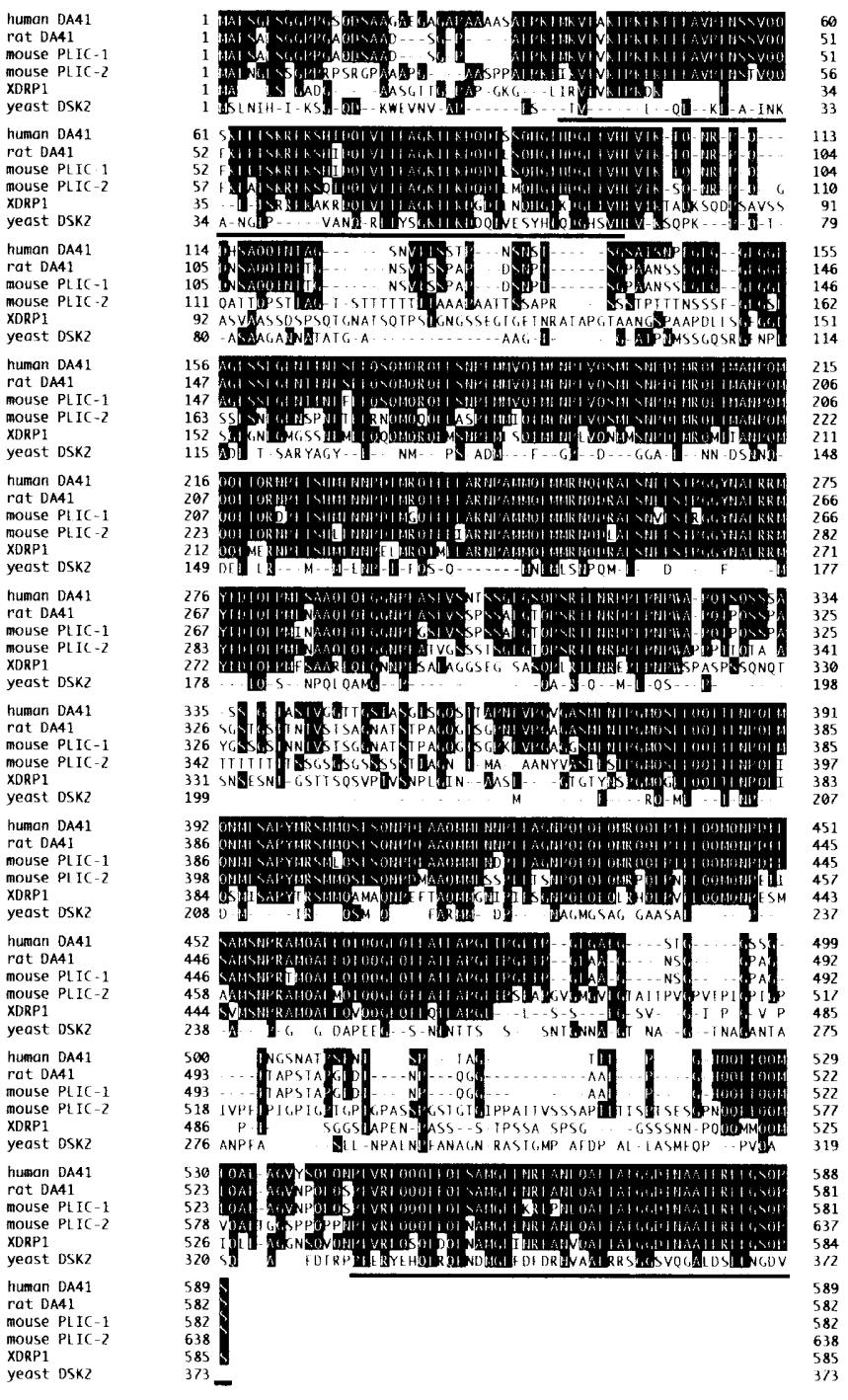

Fig. 2. The amino acid sequence alignment of human DA41 with rat DA41 (accession no. D87950) and the other previously reported DA41-related proteins; mouse PLIC-1 (accession no. AF177345), mouse PLIC-2 (accession no. AF177346), frog XDRP1 (accession no. AB030502), and yeast DSK2 (accession no. L40587). Identical amino acid residues are printed in white type on black background. Gaps are introduced to maximize the alignment. N-terminal UBQ and Cterminal UBA regions are underlined 
interact with the cytoplasmic tail of integrin-associated protein (IAP) and mediated the interaction between IAP and vimentin-containing intermediate filaments (Wu et al. 1999). XDRP1 was associated with the amino-terminal region of cyclin A and inhibited the degradation of cyclin A (Funakoshi et al. 1999). Overproduction of DSK2 induced a mitotic defect with short spindle (Biggins et al. 1996). DA41 and each DA41-related protein share evolutionarily conserved amino-terminal and carboxy-terminal regions which are homologous to ubiquitin (UBQ domain) and ubiquitinassociated region (UBA domain), respectively (Wu et al. 1999). Human DA41 was $94 \%, 88 \%, 58 \%$, and $37 \%$ identical to the UBQ domains of PLIC-1, PLIC-2, XDRP1, and DSK2, respectively. Similarly, human DA41 exhibited $96 \%$, $98 \%, 91 \%$, and $38 \%$ sequence identity to the UBA domains of PLIC-1, PLIC-2, XDRP1, and DSK2, respectively.

We analyzed the expression of the human $D A 41$ gene in adult tissues. Human RNA master blot (Clontech, Palo Alto, CA, USA) was hybridized under the conditions rec- ommended by a manufacturer. A random primer-labeled 3 '-untranslated region of human $D A 41$ cDNA was used as a probe in this experiment. As shown in Fig. 3a, human RNA master blot analysis revealed the ubiquitous expression of the $D A 41$ gene. Abundant expression was detected in adult pituitary gland, adrenal gland, kidney, thymus, and placenta. Ubiquitous expression of the $D A 41$ gene was also observed in adult rat tissues (Ozaki et al. 1997). Similarly, PLIC-1 and PLIC-2 were expressed in most mouse tissues and cell types (Wu et al. 1999).

In order to determine the chromosomal location of $D A 41$, a human PAC library was screened with PCR, using primers specific for $D A 41$ (5'-ACTGCATGCATCACTTCTGC-3') and (5'-TGGACAGATGCAGGACAAAA$\left.3^{\prime}\right)$. A single PAC clone, which was confirmed to contain $D A 41$ by Southern blot hybridization, was obtained. This PAC clone was then labeled with biotin and used for direct R-banding fluorescence in situ hybridization (FISH) (Takahashi et al. 1990, 1991). As shown in Fig. 3b, twin
Fig. 3. a The tissue distribution of $D A 41$ was examined using human RNA master blot (Clontech). The filter was hybridized with a radio-labeled cDNA probe encoding the $3^{\prime}$ untranslated region of human $D A 41$ under the conditions recommended by the manufacturer. b Fluorescence in situ hybridization (FISH) mapping of the $D A 41$ gene to $9 \mathrm{q} 21.2-\mathrm{q} 21.3$. A PAC clone containing the $D A 41$ gene was used for the chromosomal localization of $D A 41$ by FISH. Metaphase spreads prepared from normal peripheral blood lymphocytes were hybridized with a biotin-labeled probe. Arrow indicates the positive signals on 9q21.2-q21.3 $\mathbf{a}$
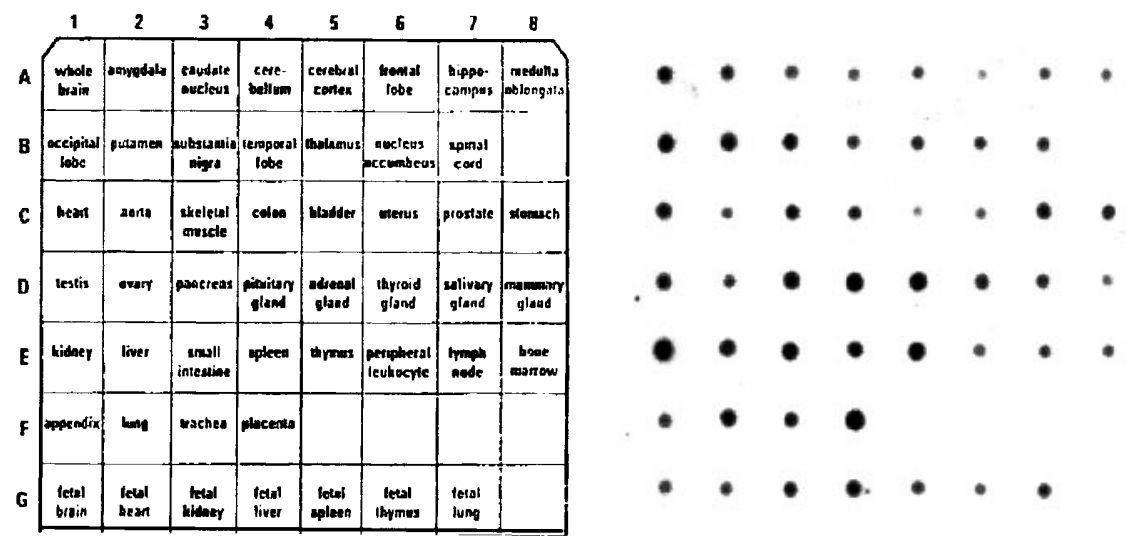

b

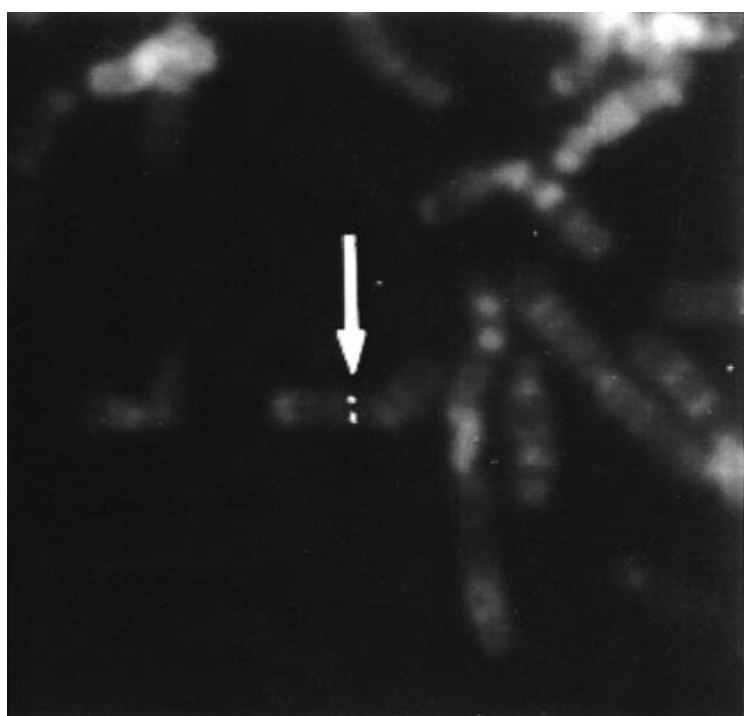


signals were observed on the $\mathrm{q} 21.2-\mathrm{q} 21.3$ band of chromosome 9.

Funakoshi et al. (1999) have found that frog XDRP1, which exhibits extensive homology with DA41, can associate with cyclin A-CDK complex. Overproduction of XDRP1 inhibited the degradation of cyclin A and blocked cell division. These observations strongly suggest that DA41 and XDRP1 could share a similar function in cell cycle regulation. It is intriguing to note that the expression of $D A 41$ was regulated in a cell cycle-dependent manner in synchronized 3 Y1 rat fibroblasts (Ozaki et al. 1997). Recently, two candidate tumor suppressor loci for bladder cancer were identified on chromosome 9; 9p21-q21 and distal chromosome 9q (Simoneau et al. 1996). In addition, the proximal region of chromosome $9 \mathrm{q}$ was deleted in bladder cancer (Bernues et al. 1993). Because DA41 was mapped within or near the candidate locus for bladder cancer, $D A 41$ may be involved in bladder carcinogenesis. To test this possibility, information on structural alteration(s) within $D A 41$ in primary bladder cancers is necessary.

\section{References}

Bernues M, Casadevall C, Miro R, Caballin MR, Gelabert A, Egozcue J (1993) A case of transitional cell carcinoma of the bladder with a del(9)(q11q21.2). Cancer Genet Cytogenet 69:76-77

Biggins S, Ivanovska I, Rose MD (1996) Yeast ubiquitin-like genes are involved in duplication of the microtuble organizing center. J Cell Biol 133:1331-1346

Funakoshi M, Geley S, Hunt T, Nishimoto T, Kobayashi H (1999) Identification of XDRP1; a Xenopus protein related to yeast Dsk2p binds to the N-terminus of cyclin $\mathrm{A}$ and inhibits its degradation. EMBO J 18:5009-5018

Hsu DR, Economides AN, Wang X, Eimon PM, Harland RM (1998) The Xenopus dorsalizing factor Gremlin identifies a novel family of secreted proteins that antagonize BMP activities. Mol Cell 1:673-683
Kozak M (1987) An analysis of 5'-noncoding sequence from 699 vertebrate messenger RNAs. Nucleic Acids Res 15:8125-8148

Nakamura Y, Ozaki T, Nakagawara A, Sakiyama S (1997) A product of $D A N$, a novel candidate tumor suppressor gene, is secreted into culture medium and suppresses DNA synthesis. Eur J Cancer 33:1986-1990

Ozaki T, Sakiyama S (1993) Molecular cloning and characterization of a cDNA showing negative regulation in v-src-transformed $3 \mathrm{Y} 1$ rat fibroblasts. Proc Natl Acad Sci USA 90:2593-2597

Ozaki T, Sakiyama S (1994) Tumor-suppressive activity of N03 gene product in v-src-transformed rat 3Y1 fibroblasts. Cancer Res 54:646648

Ozaki T, Ma J, Takenaga K, Sakiyama S (1996) Cloning of mouse $D A N$ cDNA and its down-regulation in transformed cells. Jpn J Cancer Res 87:58-61

Ozaki T, Hishiki T, Toyama Y, Yuasa S, Nakagawara A, Sakiyama S (1997) Identification of a new cellular protein that can interact specifically with DAN. DNA Cell Biol 16:985-991

Pearce JJH, Penny G, Rossant J (1999) A mouse Cerberus/Dan-related gene family. Dev Biol 209:98-110

Simoneau AR, Spruck CH, Gonzalez-Zulueta M, Gonzalgo ML, Chan MF, Tsai YC, Dean M, Steven K, Horn T, Jones PA (1996) Evidence for two tumor suppressor loci associated with proximal chromosome $9 \mathrm{p}$ to $\mathrm{q}$ and distal chromosome $9 \mathrm{q}$ in bladder cancer and the initial screening for GAS1 and PTC mutations. Cancer Res 56:5039-5043

Stanley E, Biben C, Kotecha S, Fabri L, Tajbakhsh S, Wang C-C, Hatzistavrou T, Roberts B, Drinkwater C, Lah M, Buckingham M, Hilton D, Nash A, Mohun T, Harvey RP (1998) DAN is a secreted glycoprotein related to Xenopus cerberus. Mech Dev 77:173-184

Takahashi E, Hori T, O'Connell P, Leppert M, White R (1990) R-banding and nonisotopic in situ hybridization: Precise localization of the human type II collagen gene (COL2A1). Hum Genet 86:14-16

Takahashi E, Yamauchi M, Tsuji H, Hitomi A, Meuth M, Hori T (1991) Chromosome mapping of the human cytidine-5' -triphosphate synthetase (CTPS) gene to band $1 \mathrm{p} 34.1-\mathrm{p} 34.3$ by fluorescence in situ hybridization. Hum Genet 88:119-121

Topol LZ, Marx M, Laugier D, Bogdanova NN, Boubnov NV, Clausen PA, Calothy G, Blair DG (1997) Identification of $\mathrm{drm}$, a novel gene whose expression is suppressed in transformed cells and which can inhibit growth of normal but not transformed cells in culture. Mol Cell Biol 17:4801-4810

Wu A-L, Wang J, Zheleznyak A, Brown EJ (1999) Ubiquitin-related proteins regulate interaction of vimentin intermediate filaments with the plasma membrane. Mol Cell 4:619-625 